\title{
Synthetic Aperture Radar Image Background Clutter Fitting Using SKS + MoM-Based $G^{0}$ Distribution
}

\author{
Zhengwei Zhu, ${ }^{1,2,3}$ Jianjiang Zhou, ${ }^{2}$ and Hongyu Chu ${ }^{1,3}$ \\ ${ }^{1}$ School of Information Engineering, Southwest University of Science and Technology, Mianyang 621010, China \\ ${ }^{2}$ College of Information Science and Technology, Nanjing University of Aeronautics and Astronautics, Nanjing 210016, China \\ ${ }^{3}$ Robot Technology Used for Special Environment Key Laboratory of Sichuan Province, \\ Southwest University of Science and Technology, Mianyang, Sichuan 621010, China
}

Correspondence should be addressed to Zhengwei Zhu; zhuzwin@163.com

Received 10 April 2015; Revised 18 August 2015; Accepted 6 September 2015

Academic Editor: Dan Simon

Copyright (C) 2015 Zhengwei Zhu et al. This is an open access article distributed under the Creative Commons Attribution License, which permits unrestricted use, distribution, and reproduction in any medium, provided the original work is properly cited.

$G^{0}$ distribution can accurately model various background clutters in the single-look and multilook synthetic aperture radar (SAR) images and is one of the most important statistic models in the field of SAR image clutter modeling. However, the parameter estimation of $G^{0}$ distribution is difficult, which greatly limits the application of the distribution. In order to solve the problem, a fast and accurate $G^{0}$ distribution parameter estimation method, which combines second-kind statistics (SKS) technique with Freitas' method of moment (MoM), is proposed. First we deduce the first and second second-kind characteristic functions of $G^{0}$ distribution based on Mellin transform, and then the logarithm moments and the logarithm cumulants corresponding to the above-mentioned characteristic functions are derived; finally combined with Freitas' method of moment, a simple iterative equation which is used for estimating the $G^{0}$ distribution parameters is obtained. Experimental results show that the proposed method has fast estimation speed and high fitting precision for various measured SAR image clutters with different resolutions and different number of looks.

\section{Introduction}

SAR image background clutter statistic modeling [1-6] is one of the basic subjects in the SAR image interpretation such as SAR target detection and discrimination. The research on the subject has important theoretical value and wide application prospects in the automatic target detection and discrimination in high-resolution SAR images [7, 8]. Among the SAR image clutter statistic models, the modeling ability of $K$ distribution is good for the heterogeneous clutters, but it cannot fit well for extremely heterogeneous clutters [8]. $\beta^{\prime}$ distribution has a good modeling ability for homogeneous, heterogeneous, and extremely heterogeneous clutters, but the distribution only can be applied to single-look SAR images [9]. $G^{0}$ distribution, which is proposed by Frery et al. [10], not only has very strong modeling capability for the clutters with wide intensity range and different intensity uniformity but also has very good compatibility for most of clutter models. The distribution can fit various background clutter regions in the single-look and multilook SAR images well and so far it is one of the optimal statistic models in the SAR image background clutter fitting [11], but the distribution also has the defect that the parameter estimation is difficult, which has become a main technical bottleneck in a practical application of the distribution. At present, the typical $G^{0}$ distribution parameter estimation methods mainly have Freitas' moment method [1] and Frery's moment estimation method [10]. The shape and scale parameters of $G^{0}$ distribution are estimated by using the first-order and second-order moments in Freitas' method, and Frery's method realizes the parameter estimation by using $1 / 2$ - and 1/4-order moments. The former of the above-mentioned two methods is simpler; the latter needs to be solved by using a numerical method and the calculation cost is larger. But both above-mentioned methods have several common disadvantages: (1) a priori knowledge of the SAR image needs to be used for obtaining the parameter equivalent number of looks (ENL) [12], (2) due to the constraint of convergence condition, the full range estimation of $G^{0}$ distribution parameters cannot be realized $[13,14]$, and (3) for very 
homogeneous clutter, the parameter ENL usually is greater than one; the estimation results of the above-mentioned two methods might be wrong $[13,14]$. These defects result in that the parameters of $G^{0}$ distribution obtained by the above-mentioned two methods are not very accurate and the fitting precision of $G^{0}$ distribution for the clutters is declined. Theoretically the maximum likelihood estimation (MLE) can also be used for estimating the parameters of $G^{0}$ distribution, but its equations that are used for estimating the distribution parameters are highly nonlinear, which might cause a sharp rise in the calculation and even a wrong estimated result, so the method is rarely applied in the parameter estimation of $G^{0}$ distribution. In order to solve the $G^{0}$ distribution parameter estimation problem, by studying and analyzing the modeling principle of $G^{0}$ distribution, a new, fast, and accurate $G^{0}$ distribution parameter estimation method, which combines second-kind statistics (SKS) technique with Freitas' method of moment (MoM), is proposed in this paper. The proposed method starts from Mellin transform, by deducing the first and second second-kind characteristic functions of $G^{0}$ distribution, the logarithm moments (log-moments), and logarithm cumulants (log-cumulants) corresponding to the characteristic functions, and then, combining with Freitas' moment estimation method, a simple iterative equation, which is used for estimating the $G^{0}$ distribution parameters, namely, the equivalent number of looks (ENL), the shape parameter, and the scale parameter, is obtained ultimately. The method not only solves the problems that "the MoM cannot realize the full range estimation of $G^{0}$ distribution parameters" and "the MLE needs a long iterative time and the accuracy of the iteration is poor," but, more importantly, the method considers the parameter ENL as an unknown estimated parameter like the shape and scale parameters, which can ensure that $G^{0}$ distribution has a high fitting precision for the clutters. Finally, the performance of the proposed method is compared and evaluated by using the measured SAR images with different resolutions and different number of looks. Experimental results show that not only the ENL, shape, and scale parameters can be more quickly and accurately estimated by using the proposed method but also the estimated ENL, shape, and scale parameters are more reasonable than other methods. The proposed method has a high fitting precision for the measured SAR image clutter regions with wide changes in the uniformity.

\section{New Parameter Estimation Method for $G^{0}$ Distribution}

For a SAR intensity image, the expression of $G^{0}$ distribution is

$$
f_{X_{I}}(x)=\frac{n^{n} \Gamma(n-\alpha) x^{n-1}}{\gamma^{\alpha} \Gamma(n) \Gamma(-\alpha)(\gamma+n x)^{n-\alpha}},
$$

where $-\alpha, \gamma, n$, and $x>0 . x$ is the intensity of pixels. $n$ is the equivalent number of looks. $\alpha$ is the shape parameter, which essentially reflects the uniformity of a measured clutter region. The parameter $\alpha \in(-\infty, 0)$ indicates that $G^{0}$ distribution can model various clutter regions with wide uniformity changes. The bigger the parameter $|\alpha|$ is, the more uniform the corresponding clutter region is. $\gamma$ is the scale parameter, which is related to the average energy of a measured clutter region. The greater the parameter $\gamma$ is, the greater the average energy of the corresponding clutter region is.

In order to fundamentally solve the parameter estimation problem of $G^{0}$ distribution, a new estimation method of the $G^{0}$ distribution parameters $n, \alpha$, and $\gamma$ will be proposed in the following. What needs to be pointed out is that all the parameters are estimated based on the intensity form of $G^{0}$ distribution in the following discussion.

2.1. Second-Kind Statistics Method. By analyzing the modeling principle of $G^{0}$ distribution, we found that $G^{0}$ distribution derives from the speckle model and also integrates with some characteristics of the multiplicative model. We know that the speckle noise may be considered as Mellin convolution in the Mellin transform-based second-kind statistics [4-6], which coincides with the SAR image multiplicative model, so it may be expected that, by imitating the solving ideas that "the first and second second-kind characteristic functions may be generated by Fourier transform and then the cumulants can be obtained by taking the derivative of the characteristic functions at zero" [15], we can derive a new parameter estimation method of $G^{0}$ distribution by using Mellin transform, which can greatly reduce the difficulty of $G^{0}$ distribution parameter estimation.

For a function $f(x)$ with the domain $R^{+}$, the following integral is defined as Mellin transform [4-6]:

$$
\phi(s) \triangleq M[f(x)](s) \triangleq \int_{0}^{\infty} x^{s-1} f(x) d x
$$

where the two equals signs with a Delta over them denote a definition formula of Mellin transform given behind them. We know that the domain of the probability density function of a SAR intensity image just is $R^{+}$, so Mellin transform may be applied to analyze the SAR intensity image.

Substituting formula (1) into formula (2), we have

$$
\phi(s)=\frac{n^{n} \Gamma(n-\alpha)}{\gamma^{n} \Gamma(n) \Gamma(-\alpha)} \int_{0}^{\infty} \frac{x^{s+n-2}}{(1+(n / \gamma) x)^{n-\alpha}} d x
$$

According to formula (3) and [16], we may obtain

$$
\phi(s)=\left(\frac{\gamma}{n}\right)^{s-1} \frac{\Gamma(n+s-1) \Gamma(-\alpha-(s-1))}{\Gamma(n) \Gamma(-\alpha)} .
$$

By carefully observing formula (4), we can find that if we take the logarithm on both sides of the formula, this will greatly simplify the computation:

$$
\begin{aligned}
\varphi(s) & \triangleq \ln (\phi(s)) \\
& =\ln \left[\left(\frac{\gamma}{n}\right)^{s-1} \frac{\Gamma(n+s-1) \Gamma(-\alpha-(s-1))}{\Gamma(n) \Gamma(-\alpha)}\right]
\end{aligned}
$$




$$
\begin{aligned}
= & (s-1) \ln \left(\frac{\gamma}{n}\right)+\ln \Gamma(n+s-1) \\
& +\ln \Gamma(-\alpha-(s-1))-\ln \Gamma(n)-\ln \Gamma(-\alpha),
\end{aligned}
$$

where $\varphi(s)$ is called the second second-kind characteristic function corresponding to $G^{0}$ distribution. By taking the derivative of the function at $s=1$, we have

$$
\left.\widetilde{c}_{k} \triangleq \frac{d^{k} \varphi(s)}{d s^{k}}\right|_{s=1},
$$

where $\widetilde{c}_{k}, k=1,2, \ldots$, are called log-cumulants.

Substituting formula (5) into formula (6), we can obtain the log-cumulants corresponding to $G^{0}$ distribution:

$$
\begin{aligned}
& \widetilde{c}_{1}=\ln \left(\frac{\gamma}{n}\right)+\psi(n)-\psi(-\alpha) \\
& \widetilde{\mathcal{c}}_{k}=\psi(k-1, n)+(-1)^{k} \psi(k-1,-\alpha), \quad k \geq 2,
\end{aligned}
$$

where $\psi(z)=d \ln \Gamma(z) / d z$ is called digamma function, which is the first-order derivative of logarithm gamma function. $\psi(k-1, z)=d^{k} \ln \Gamma(z) / d z^{k}$ is called $(k-1)$-order polygamma function. Obviously, formulas (7) of log-cumulants are concise and easy to be computed. More fortunately, thereto the polygamma function is a monotonic function, so formulas (7) are very suitable to be used for estimating parameters $\alpha$, $\gamma$, and $n$ of $G^{0}$ distribution.

Instead of the log-cumulants, usually the sample logcumulants are used in a practical application. Supposing that $x_{1}, x_{2}, \ldots$, and $x_{N}$ are grayscale observations of $N$ sample pixels of a certain clutter region, then the formulas of sample log-cumulants may be expressed as

$$
\begin{aligned}
& \widetilde{c}_{1}=\frac{1}{N} \sum_{i=1}^{N}\left[\ln \left(x_{i}\right)\right] \\
& \widetilde{c}_{k}=\frac{1}{N} \sum_{i=1}^{N}\left[\left(\ln \left(x_{i}\right)-\widetilde{c}_{1}\right)^{k}\right], \quad k \geq 2 .
\end{aligned}
$$

Combining formula (7) with formula (8), we can obtain the ultimate estimation equations of $G^{0}$ distribution parameters $n, \gamma$, and $\alpha$ :

$$
\begin{aligned}
\ln \left(\frac{\gamma}{n}\right)+\psi(n)-\psi(-\alpha) & =\widetilde{c}_{1} \\
\psi(1, n)+\psi(1,-\alpha) & =\frac{1}{N} \sum_{i=1}^{N}\left[\left(\ln \left(x_{i}\right)-\widetilde{c}_{1}\right)^{2}\right] \\
\psi(2, n)-\psi(2,-\alpha) & =\frac{1}{N} \sum_{i=1}^{N}\left[\left(\ln \left(x_{i}\right)-\widetilde{c}_{1}\right)^{3}\right],
\end{aligned}
$$

where (9) are called the second-kind statistics (SKS) estimator and the corresponding parameter estimation method is called the SKS method.

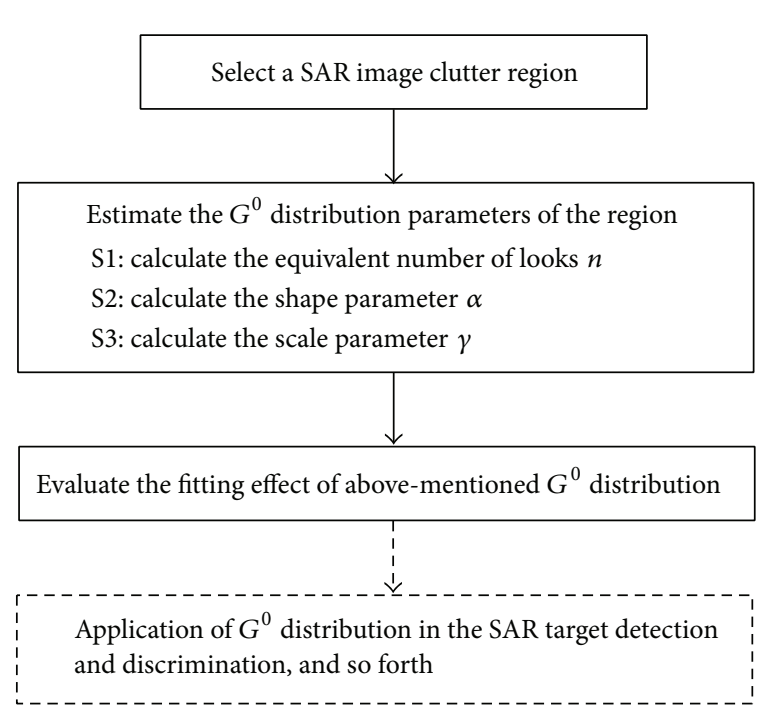

FIGURE 1: The processing flow of the proposed method.

2.2. Advantages and Disadvantages of the SKS Method. By analyzing the above-mentioned estimation method of $G^{0}$ distribution parameters and compared with the moment estimator and the maximum likelihood estimator, we can draw the following conclusions.

(1) The convergence condition of obtaining formula (4) from formula (3) by integral is

$$
\begin{aligned}
n+s-1 & >0 \\
-\alpha & >s-1 \\
n & >\alpha
\end{aligned}
$$

$$
\alpha<0, n>0 \text {. }
$$

The purpose of obtaining formula (4) by integral is to get the log-cumulants corresponding to $G^{0}$ distribution. We can see from formula (6) that the log-cumulants are only concerned with the derivative of $\varphi(s)$ at the point $s=1$. When $s=$ 1 , the convergence condition (10) is obviously satisfied. So the SKS estimator successfully solves the problem that "the moment estimator cannot realize the full range estimate of $G^{0}$ distribution parameters due to the constraint of convergence condition," which can ensure that $G^{0}$ distribution has a high fitting precision for the clutter regions with wide uniformity changes.

(2) The moment estimator and the maximum likelihood estimator usually use a priori knowledge of a SAR image to obtain the ENL parameter $n$ or take the ISNR (inherent signal to noise ratio) $[13,17]$ as the ENL parameter $n$ of a SAR image. In the above-mentioned two methods to obtain parameter $n$, the partial correlation between the samples that participate in average processing will bring the bias to the estimated parameter $n$, which will result in the decline of the fitting precision. But the ENL parameter $n$ is considered as an unknown estimated parameter like the shape and scale parameters in the SKS estimator, so it solves the problem of the estimation bias of the ENL parameter $n$ in the moment estimator and the maximum likelihood estimator well. 


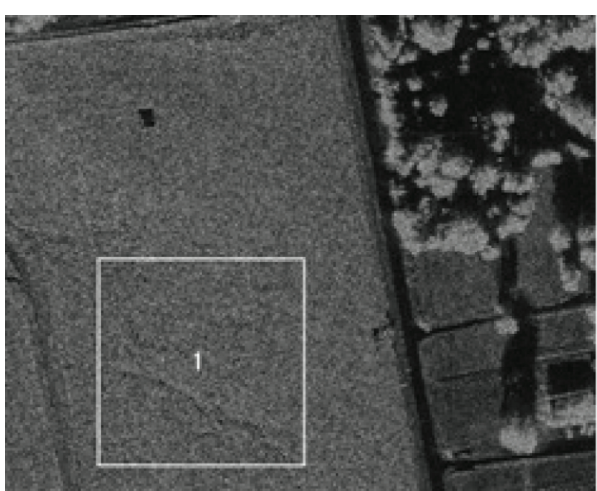

(a) Region 1

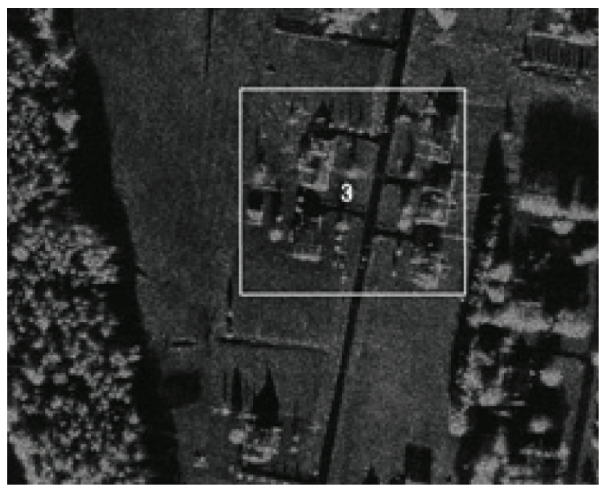

(c) Region 3

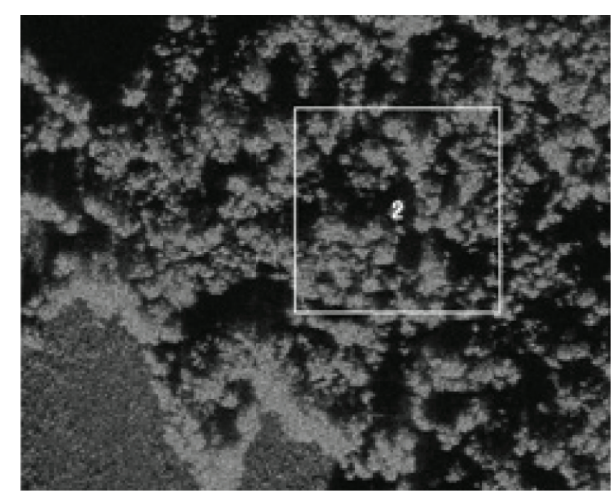

(b) Region 2

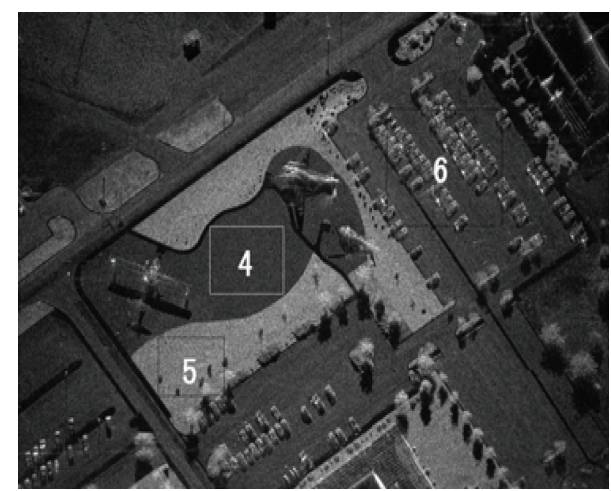

(d) Regions 4, 5, and 6

FIgURE 2: The SAR images used in our experiment.

(3) We can easily see that when $k \geq 2$, it has a strong regularity between the last two equations of (9) and moreover the polygamma functions that they contain are monotonic, so the solution of the equations, namely, the estimates of the shape, scale, and ENL parameters, can be quickly and accurately obtained by using a numerical iteration method, which overcomes the defects that the maximum likelihood estimator needs a long iteration time and has a poor iteration accuracy.

(4) When a clutter region is very homogeneous, the estimates of $G^{0}$ distribution parameters are very difficult to be obtained by using the SKS method.

Usually, a good fitting effect can be achieved by the SKS method. But in practice we found that when a clutter region is very homogeneous, the solution of (9) is very difficult to be obtained. As mentioned above, it has a strong regularity between the last two equations of (9), and moreover the function $\psi(1, x)$ is monotonically decreasing and is close to zero with increasing of $x$ in its definition domain and the function $\psi(2, x)$ is monotonically increasing and is close to zero with increasing of $x$ in its definition domain, so the solution of the equations, namely, the estimates of parameters $n, \alpha$, and $\gamma$, can be quickly and accurately determined by using a numerical method. For (9), let

$$
\begin{aligned}
& f_{1}(x)=\psi^{-1}\left[1, \widetilde{k}_{2}-\psi(1,-x)\right] \\
& f_{2}(x)=\psi^{-1}\left[2, \widetilde{k}_{3}-\psi(2,-x)\right],
\end{aligned}
$$

where $\psi^{-1}(k, x)$ represents the inverse function of $\psi(k, x)$. It may be proved that the function $f_{1}(x)$ is monotonically decreasing with increasing of $x$ and the function $f_{2}(x)$ is monotonically increasing with increasing of $x$. So in theory, the intersection of the curves $f_{1}(x)$ and $f_{2}(x)$ just is the estimates of parameters $n$ and $\alpha$ that we want to obtain, and then the estimate of parameter $\gamma$ can be obtained by substituting the estimates of parameters $n$ and $\alpha$ into (9). However, when a clutter region is very homogeneous, the curves $f_{1}(x)$ and $f_{2}(x)$ would never intersect; that is to say, the solution of (9) is very difficult to be obtained. By analyzing (9) and formula (11), we can find that when a clutter region is very homogeneous, the value of $|\alpha|$ will be very large and the functions $\psi(1, x)$ and $\psi(2, x)$ will approach zero. When $|\alpha| \rightarrow \infty$, both $f_{1}(x) \rightarrow \psi^{-1}\left(1, \widetilde{k}_{2}\right)$ and $f_{2}(x) \rightarrow \psi^{-1}\left(2, \widetilde{k}_{3}\right)$ are constants; if $\psi^{-1}\left(1, \widetilde{k}_{2}\right)>\psi^{-1}\left(2, \widetilde{k}_{3}\right)$, then the curves $f_{1}(x)$ and $f_{2}(x)$ would never intersect; that is to say, the solution of (9) is very difficult to be obtained.

So we can conclude from the above-mentioned analysis that when a clutter region is very homogeneous, the estimates of $G^{0}$ distribution parameters will be difficult to be obtained by the SKS method. It is this reason that prompts us to study a new $G^{0}$ distribution parameter estimation method.

2.3. New SKS + MoM Method. The ENL $n$ is considered as a parameter to be estimated in the new method. Combining the moment estimation method proposed by Freitas and the first-order logarithm cumulant that is derived from Mellin 
transform, a new $G^{0}$ distribution parameter estimation method may be obtained as follows:

$$
\begin{aligned}
& \ln \left(\frac{\gamma}{n}\right)+\psi(n)-\psi(-\alpha)=\frac{1}{N} \sum_{i=1}^{N}\left[\ln \left(x_{i}\right)\right] \\
& \gamma=-(\alpha+1) E(x) \\
& \alpha=\frac{2 n E\left(x^{2}\right)-(n+1) E^{2}(x)}{(n+1) E^{2}(x)-n E\left(x^{2}\right)} .
\end{aligned}
$$

Using the first-order origin moment of $G^{0}$ distribution, we can obtain

$$
\frac{\gamma}{n}=\frac{E\left(x^{2}\right) E(x)}{n E\left(x^{2}\right)-(n+1) E^{2}(x)} .
$$

Substituting formula (13) and the third formula of (12) into the first equation of (12) and simultaneously considering the constraints $\gamma / n>0,-\alpha>0$, and $n>0$, the solution of $n$ can be obtained by a numerical method. Substituting the solution $n$ into the third formula of (12), we can get the numerical solution of $\alpha$. And finally substituting solution $\alpha$ into the second formula of (12), we can get the numerical solution of $\gamma$. So parameters $n, \alpha$, and $\gamma$ of $G^{0}$ distribution are obtained quickly and accurately by using the new method. The new method only uses first-order and second-order origin moments and a first-order log-cumulant. In addition, we can see in the following experiment that the new method is more robust than the SKS method.

What needs to be pointed out is that just as Freitas' moment method the shape parameter $\alpha$ in (12) still exists in the constraint $\alpha<-2$, which limits the application of the new method in the fitting of extremely heterogeneous clutters. But the main purpose of the new method is to solve the $G^{0}$ distribution parameter estimation problem when the clutters are very homogeneous (at this time, $\alpha \ll 0$ ) and the SKS method is not applicable. When the clutters are so extremely heterogeneous that their actual distribution parameter $\alpha>$ -2 , we still use the SKS method to estimate the parameters of $G^{0}$ distribution.

The proposed SKS + MoM method not only has the advantage of the SKS method that can quickly and accurately estimate the parameters of $G^{0}$ distribution but also overcomes the disadvantage of the SKS and MoM methods that when a clutter region is very homogeneous, both the SKS method and the MoM method are not applicable.

\section{Experimental Results and Analysis}

The processing flow of the proposed method is shown in Figure 1.

At present, most of the researchers segment an image into different clutter regions according to land cover object types. So we also find the initial appropriate segmentation of an image into regions with locally homogeneous clutter according to the principle that the homogeneous clutter region contains the same land cover type in the following experiments. Figure 2 shows three MSTAR_PUBLIC_CLUTTER

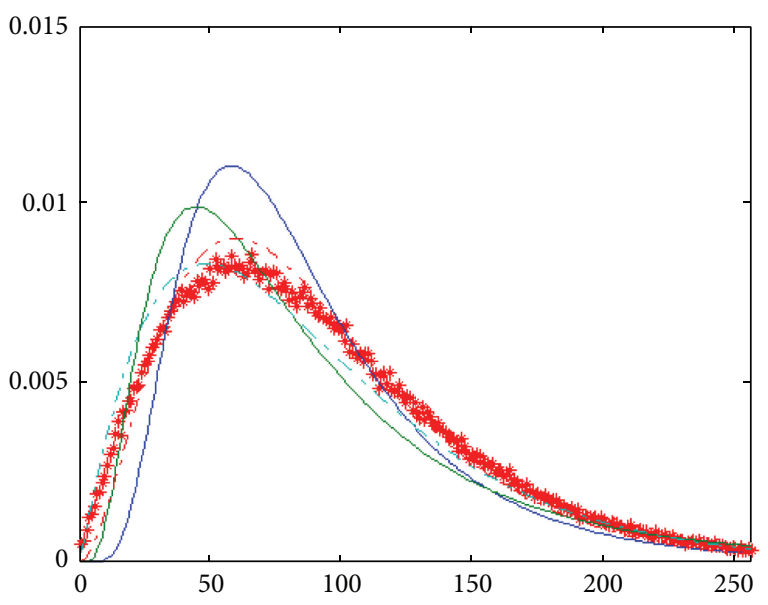



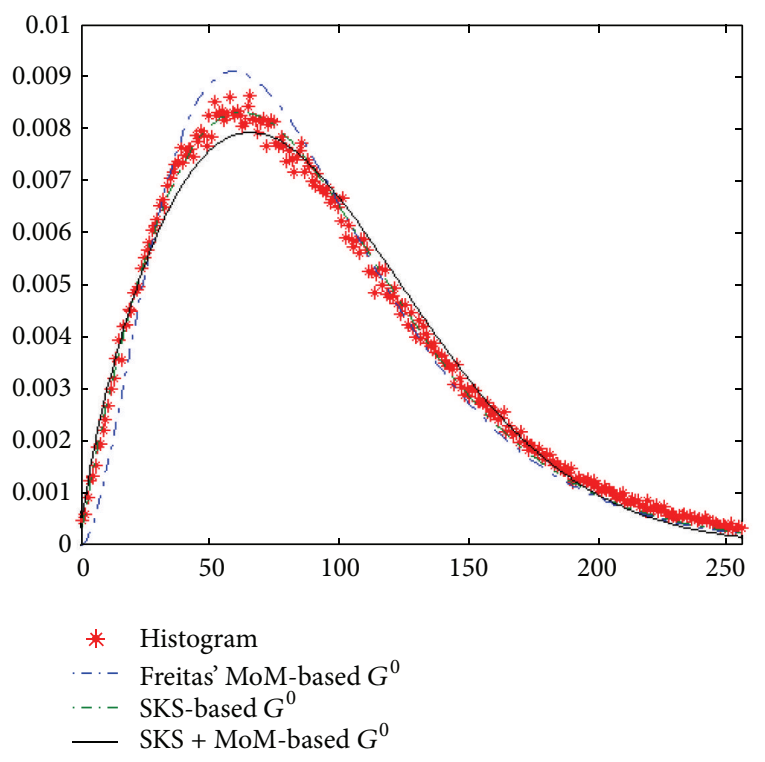

FIGURE 3: The fitting results of Region 1.

SAR images and a Ku-band SAR image provided by Sandia National Laboratories; the resolution of the former is $0.3 \mathrm{~m} \times$ $0.3 \mathrm{~m}$ and that of the latter is $0.1 \mathrm{~m} \times 0.1 \mathrm{~m}$. Six typical regions in the four SAR images are selected and used for our experiments. Among them, Region 1 is a large block of cropland and it has high clutter uniformity; Region 2 is composed of woods and shadows and it is a not very uniform clutter region; Region 3 is an extremely heterogeneous clutter region, which has very complex scenes, including a large number of heterogeneous man-made and natural clutters such as cement floors, houses, and trees; Region 4 is a block of cement floor and it is a very smooth and homogeneous clutter region; Region 5 consists of grasses and scrubby bushes and it is a relatively uniform clutter region; Region 6 is a parking lot, which contains cement floors, cars, and other objects, and it is an extremely heterogeneous clutter region. 
TABLE 1: The MSE values of nine parameter models fitting six clutter regions*.

\begin{tabular}{|c|c|c|c|c|c|c|}
\hline \multirow{2}{*}{ Parameter models } & \multicolumn{6}{|c|}{ Clutter regions } \\
\hline & 1 & 2 & 3 & 4 & 5 & 6 \\
\hline MoM-based lognormal & 17.279 & 32.756 & 11.876 & 292.04 & 242.53 & 64.221 \\
\hline MLE-based lognormal & 10.205 & 2.2485 & 5.0526 & 316.94 & 449.45 & 75.796 \\
\hline MoM-based gamma & 2.0844 & 13.792 & 6.9545 & 114.68 & 171.43 & 104.23 \\
\hline MLE-based gamma & 3.1056 & 57.116 & 8.1760 & 3682.5 & 3542.0 & 450.79 \\
\hline MoM-based $K$ & - & - & 1.6403 & - & - & - \\
\hline Freitas' MoM-based $G^{0}$ & 2.0843 & 13.792 & 6.9545 & 114.68 & 171.47 & 104.25 \\
\hline Fully parametric MoM-based $G^{0}$ & - & - & 2.1876 & - & - & 53.998 \\
\hline SKS-based $G^{0}$ & 0.3150 & 9.9094 & 2.1815 & - & - & 40.373 \\
\hline SKS + MoM-based $G^{0}$ & 0.9289 & 30.745 & 8.0961 & 38.094 & 115.80 & 39.905 \\
\hline
\end{tabular}

${ }^{*}$ Note that the MSE values in this table need to be multiplied by $10^{-10}$.

TABLE 2: The K-S test values of nine parameter models fitting six clutter regions.

\begin{tabular}{|c|c|c|c|c|c|c|}
\hline \multirow{2}{*}{ Parameter models } & \multicolumn{6}{|c|}{ Clutter regions } \\
\hline & 1 & 2 & 3 & 4 & 5 & 6 \\
\hline MoM-based lognormal & 0.0674 & 0.0955 & 0.0471 & 0.0454 & 0.0471 & 0.0403 \\
\hline MLE-based lognormal & 0.0601 & 0.0319 & 0.0315 & 0.0528 & 0.0780 & 0.0455 \\
\hline MoM-based gamma & 0.0223 & 0.0432 & 0.0331 & 0.0270 & 0.0397 & 0.0560 \\
\hline MLE-based gamma & 0.0423 & 0.1478 & 0.0564 & 0.2561 & 0.3090 & 0.1585 \\
\hline MoM-based $K$ & - & - & 0.0150 & - & - & - \\
\hline Freitas' MoM-based $G^{0}$ & 0.0223 & 0.0432 & 0.0331 & 0.0270 & 0.0396 & 0.0560 \\
\hline Fully parametric MoM-based $G^{0}$ & - & - & 0.0175 & - & - & 0.0358 \\
\hline SKS-based $G^{0}$ & 0.0101 & 0.0472 & 0.0186 & - & - & 0.0381 \\
\hline SKS + MoM-based $G^{0}$ & 0.0151 & 0.0852 & 0.0397 & 0.0203 & 0.0257 & 0.0333 \\
\hline
\end{tabular}

TABLE 3: The KL divergence values of nine models fitting six clutter regions.

\begin{tabular}{|c|c|c|c|c|c|c|}
\hline \multirow{2}{*}{ Parameter models } & \multicolumn{6}{|c|}{ Clutter regions } \\
\hline & 1 & 2 & 3 & 4 & 5 & 6 \\
\hline MoM-based lognormal & 0.3289 & 0.2730 & 0.1077 & 0.1708 & 0.2817 & 0.1059 \\
\hline MLE-based lognormal & 0.1143 & 0.0449 & 0.0349 & 0.1503 & 0.2740 & 0.1072 \\
\hline MoM-based gamma & 0.0333 & 0.0928 & 0.0585 & 0.0599 & 0.1517 & 0.1307 \\
\hline MLE-based gamma & 0.0232 & 0.3943 & 0.0807 & 1.4981 & 1.7516 & 0.5517 \\
\hline MoM-based $K$ & - & - & 0.0216 & - & - & - \\
\hline Freitas' MoM-based $G^{0}$ & 0.0333 & 0.0928 & 0.0585 & 0.0599 & 0.1517 & 0.1307 \\
\hline Fully parametric MoM-based $G^{0}$ & - & - & 0.0258 & - & - & 0.0886 \\
\hline SKS-based $G^{0}$ & 0.0055 & 0.0952 & 0.0133 & - & - & 0.0707 \\
\hline SKS + MoM-based $G^{0}$ & 0.0132 & 0.1830 & 0.0630 & 0.0081 & 0.0947 & 0.0577 \\
\hline
\end{tabular}

In the following experiments, first we adopt different estimation methods to obtain the parameters of lognormal distribution, gamma distribution, $K$ distribution, and $G^{0}$ distribution for the above-mentioned six clutter regions (for details, please see Tables 4(a) and 4(b)). Then we use the distributions to fit the gray histograms of the abovementioned six regions, respectively. Finally we adopt three metrics, namely, mean square error (MSE) [4], KolmogorovSmirnov (KS) test [18], and Kullback-Leibler (KL) divergence [19], to evaluate the fitting precisions of different parameter models for the above-mentioned six regions. Among the three metrics, the metric MSE reflects the average deviation between the hypothesis distribution and the actual distribution, the metric KS test reflects the maximum deviation between the hypothesis distribution and the actual distribution, and the metric KL divergence reflects the overall similarity between the hypothesis distribution and the actual distribution. There are both similarity and difference between the three metrics. They can evaluate the fitting precisions of the parameter models from different aspects. What needs to be specially pointed out is that the smaller the abovementioned metric values are, the better the fitting precisions are.

The fitting results of the above-mentioned parameter models for the clutter regions 1-6 are shown in Figures 3-8, respectively. The fitting precisions are given in Tables 1-3. 
TABLE 4: (a) The parameter estimation expressions of several clutter models and (b) the parameter estimation methods of $G^{0}$ distribution.

(a)

\begin{tabular}{|c|c|c|c|}
\hline Clutter models & Probability density function & Moment method & Maximum likelihood estimation \\
\hline $\begin{array}{l}\text { Lognormal } \\
\text { distribution }\end{array}$ & $\begin{array}{l}p(x) \\
=\frac{1}{\sqrt{2 \pi} \sigma x} \exp \left(-\frac{(\ln x-\mu)^{2}}{2 \sigma^{2}}\right)\end{array}$ & $\begin{array}{l}E(x)=\exp \left(\mu+\frac{1}{2} \sigma^{2}\right) \\
E\left(x^{2}\right)=\exp \left(2 \mu+2 \sigma^{2}\right)\end{array}$ & $\begin{aligned} \mu & =\frac{1}{N} \sum_{i=1}^{N} \ln x_{i} \\
\sigma^{2} & =\frac{1}{N} \sum_{i=1}^{N}\left(\ln x_{i}-\mu\right)^{2}\end{aligned}$ \\
\hline $\begin{array}{l}\text { Gamma } \\
\text { distribution }\end{array}$ & $\begin{array}{l}p(x) \\
=\frac{1}{\Gamma(n)}\left(\frac{n}{\sigma}\right)^{n} x^{n-1} \exp \left(-\frac{n x}{\sigma}\right)\end{array}$ & $\begin{array}{l}E(x)=\sigma \\
D(x)=\frac{\sigma^{2}}{n}\end{array}$ & $\begin{array}{l}\sigma=\frac{1}{N} \sum_{i=1}^{N} x_{i} \\
\psi(n)=\ln \left(\frac{n}{\sigma}\right)+\frac{1}{N} \sum_{i=1}^{N} \ln x_{i}\end{array}$ \\
\hline$K$ distribution & $\begin{array}{l}p(x) \\
=\frac{2(\alpha n x / \mu)^{(\alpha+n) / 2}}{x \Gamma(\alpha) \Gamma(n)} K_{\alpha-n}\left(2 \sqrt{\frac{\alpha n x}{\mu}}\right)\end{array}$ & $\begin{array}{l}E(x)=\mu \\
E\left(x^{2}\right)=\frac{\mu^{2}(\alpha+1)(n+1)}{\alpha n} \\
E\left(x^{3}\right)=\frac{\mu(\alpha+2)(n+2)}{\alpha n} E\left(x^{2}\right)\end{array}$ & - \\
\hline$G^{0}$ distribution & $\begin{array}{l}p(x) \\
=\frac{n^{n} \Gamma(n-\alpha) x^{n-1}}{\Gamma(n) \Gamma(-\alpha)(\gamma+n x)^{n-\alpha}}\end{array}$ & $\begin{array}{l}E(x)=-\frac{\gamma}{\alpha+1} \\
E\left(x^{2}\right)=\frac{n+1}{n} \frac{\alpha+1}{\alpha+2} E^{2}(x) \\
E\left(x^{3}\right)=\frac{n+2}{n} \frac{\alpha+1}{\alpha+3} E\left(x^{2}\right) E(x)\end{array}$ & $\begin{array}{l}\frac{\alpha}{\gamma}=(\alpha-n) \frac{1}{N} \sum_{i=1}^{N} \frac{1}{\gamma+n x_{i}} \\
\psi(n-\alpha)+\ln \gamma-\psi(-\alpha) \\
=\frac{1}{N} \sum_{i=1}^{N} \ln \left(\gamma+n x_{i}\right) \\
\ln n+1+\psi(n-\alpha)-\psi(n) \\
=\frac{1}{N} \sum_{i=1}^{N} \ln \left(\gamma+n x_{i}\right)+\frac{n-\alpha}{N} \sum_{i=1}^{N} \frac{x_{i}}{\gamma+n x_{i}} \\
\quad-\frac{1}{N} \sum_{i=1}^{N} \ln x_{i}\end{array}$ \\
\hline
\end{tabular}

(b)

Estimation method

Freitas' MoM*

Fully parametric MoM

SKS

SKS + MoM
Estimation formula

$\alpha=\frac{2 n E\left(x^{2}\right)-(n+1) E^{2}(x)}{(n+1) E^{2}(x)-n E\left(x^{2}\right)}$

$\gamma=-(\alpha+1) E(x)$

$E(x)=-\frac{\gamma}{\alpha+1}$

$E\left(x^{2}\right)=\frac{n+1}{n} \frac{\alpha+1}{\alpha+2} E^{2}(x)$

$E\left(x^{3}\right)=\frac{n+2}{n} \frac{\alpha+1}{\alpha+3} E\left(x^{2}\right) E(x)$

$\ln \left(\frac{\gamma}{n}\right)+\psi(n)-\psi(-\alpha)=\widetilde{c}_{1}=\frac{1}{N} \sum_{i=1}^{N}\left[\ln \left(x_{i}\right)\right]$

$\psi(1, n)+\psi(1,-\alpha)=\frac{1}{N} \sum_{i=1}^{N}\left[\left(\ln \left(x_{i}\right)-\widetilde{c}_{1}\right)^{2}\right]$

$\psi(2, n)-\psi(2,-\alpha)=\frac{1}{N} \sum_{i=1}^{N}\left[\left(\ln \left(x_{i}\right)-\widetilde{c}_{1}\right)^{3}\right]$

$\ln \left(\frac{\gamma}{n}\right)+\psi(n)-\psi(-\alpha)=\frac{1}{N} \sum_{i=1}^{N}\left[\ln \left(x_{i}\right)\right]$

$\gamma=-(\alpha+1) E(x)$

$\alpha=\frac{2 n E\left(x^{2}\right)-(n+1) E^{2}(x)}{(n+1) E^{2}(x)-n E\left(x^{2}\right)}$

${ }^{*} n$ is obtained by computing the equivalent number of looks ENL $=\mu^{2} / \sigma^{2}$ of image, in which $\mu$ is the mean and $\sigma^{2}$ is the variance of image pixel intensity or amplitude, respectively $[13,17]$. 

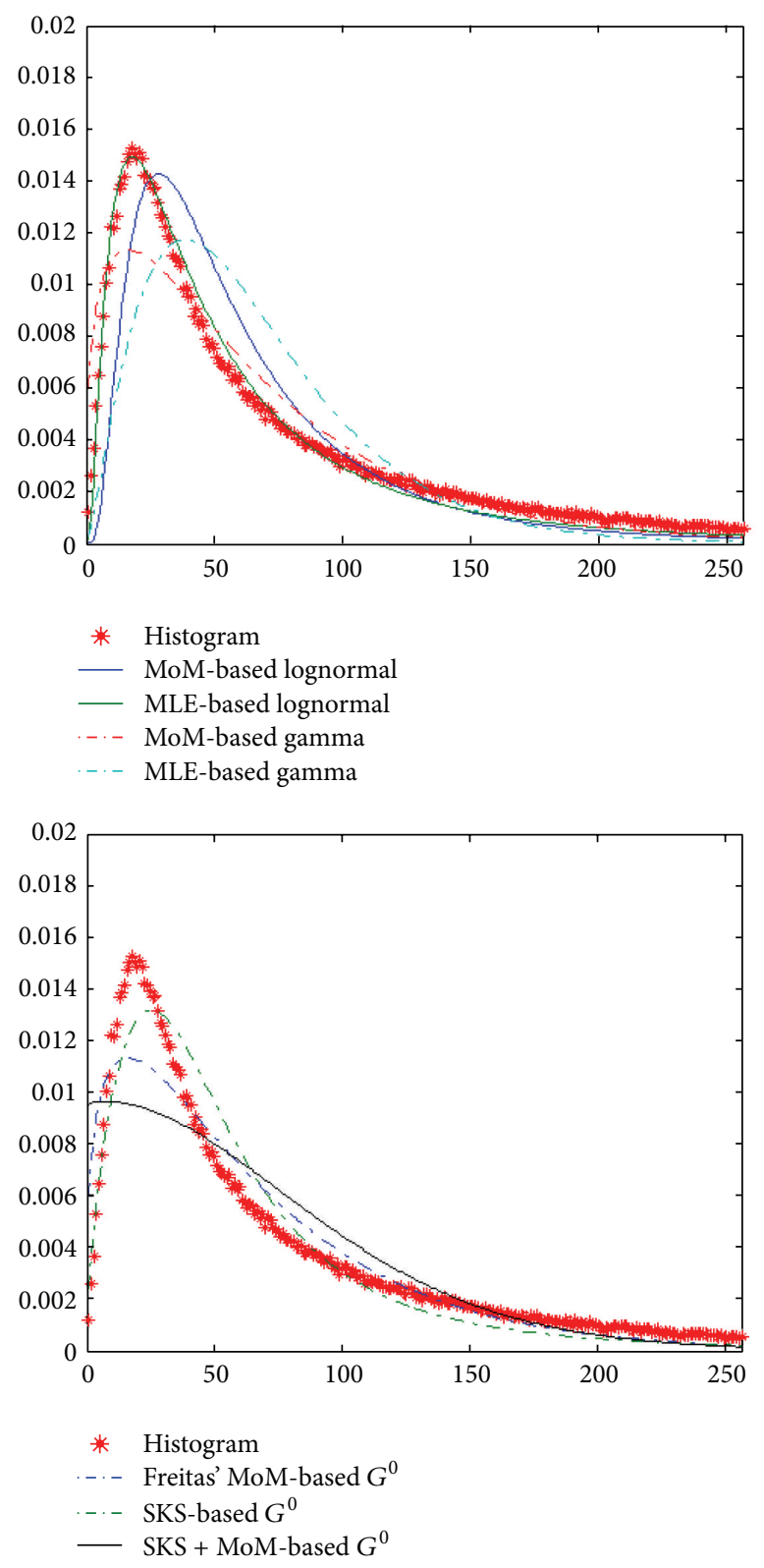

FIGURE 4: The fitting results of Region 2.

We can see from Figures 3-8 and Tables 1-3 that the MoM-based $K$ distribution and the fully parametric MoMbased $G^{0}$ distribution cannot obtain correct fitting parameter estimates for Region 1 and Region 2. For Region 3, all the distributions, including the MoM-based lognormal distribution, the MLE-based lognormal distribution, the MoM-based gamma distribution, the MLE-based gamma distribution, the MoM-based $K$ distribution, Freitas' MoM-based $G^{0}$ distribution, the fully parametric MoM-based $G^{0}$ distribution, the SKS-based $G^{0}$ distribution, and the SKS + MoM-based $G^{0}$ distribution, can obtain correct fitting parameter estimates. For Region 4 and Region 5, three methods, namely, the MoM-based $K$ distribution, the fully parametric MoM-based $G^{0}$ distribution, and the SKS-based $G^{0}$ distribution, cannot
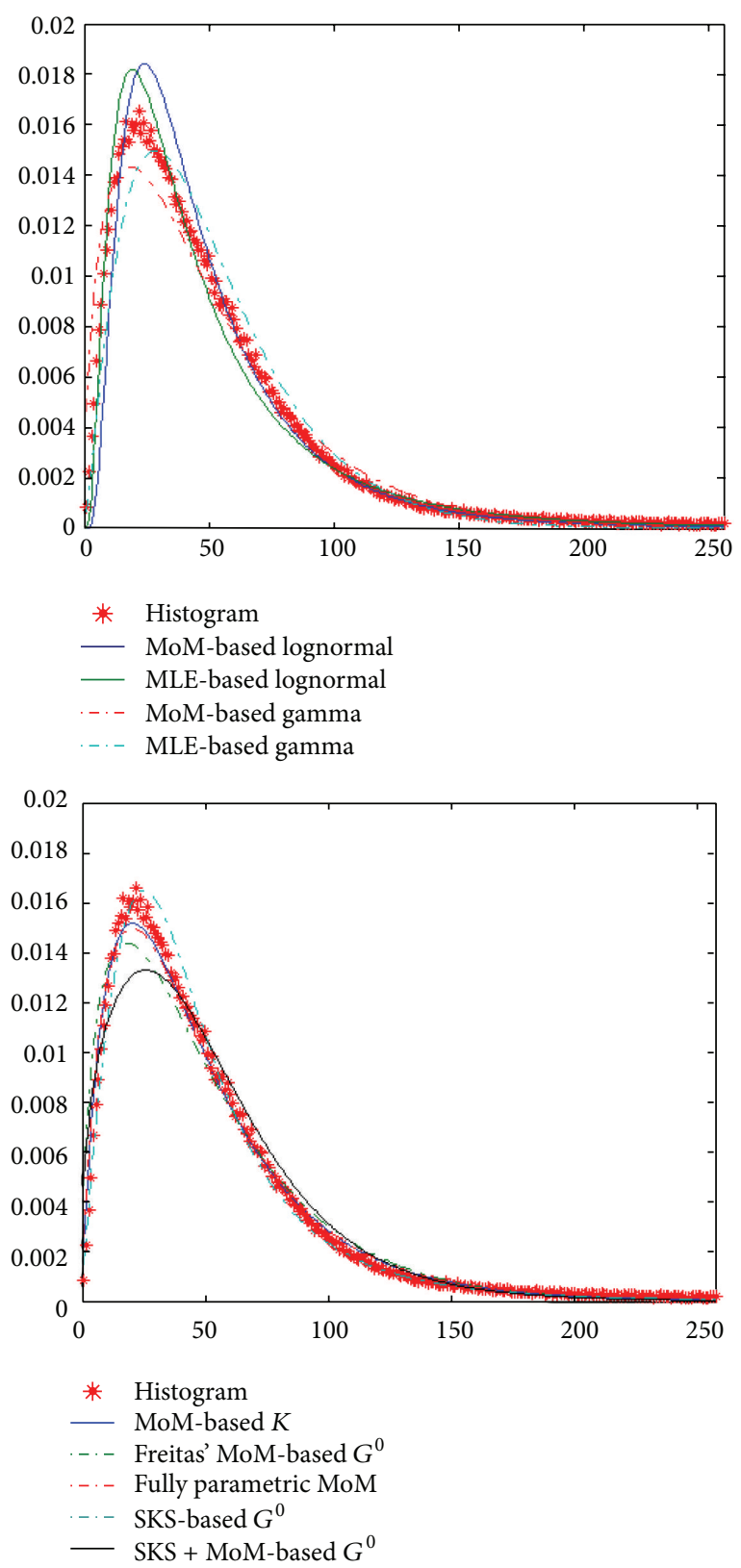

FIGURE 5: The fitting results of Region 3.

obtain correct fitting parameter estimates. For Region 6, only the MoM-based $K$ distribution cannot obtain correct fitting parameter estimates.

In addition, we can see from Figures 3-8 and Tables 1-3 the following. (1) As a whole, the fitting effects of $G^{0}$ distribution are superior to those of other distributions. (2) Assuming that $n$ is known or obtained by the given SAR image, Freitas' method, which uses the first-order and second-order moments to realize the estimation of $G^{0}$ distribution parameters $\alpha$ and $\gamma$, has relatively wide versatility, but its estimation precision is not high. (3) The SKS method has no solution for the regions where the clutters are relatively uniform. This is exactly the problem to be solved by the proposed method. (4) The proposed SKS + MoM method can 



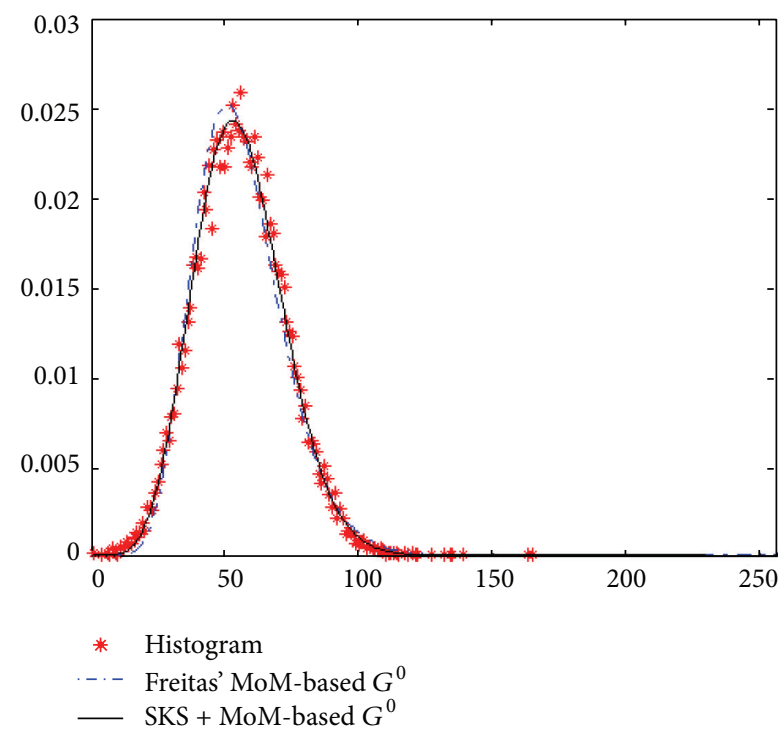

Figure 6: The fitting results of Region 4.

realize the unity of versatility and precision well. Whatever ground object clutter or SAR image resolution, the method can obtain relatively accurate parameter estimates and good fitting results. However, we also note that the fitting precisions of the proposed method are not very high for Region 2 and Region 3; it is because the clutters in the two regions are so extremely heterogeneous that the actual values of the shape parameter $\alpha$ exceed the application scope of the method $\alpha<$ -2 , which are also verified by $\alpha$ values that are estimated by the SKS method. It should be pointed out that different scenes and different regions of the same scene have different gray histograms, so the same fitting approach will have different fitting effects for different scenes and different regions of the same scene. So it is necessary for us to build a specific fitting model for a specific application.



FIGURE 7: The fitting results of Region 5.

\section{Concluding Remarks}

A SKS + MoM-based $G^{0}$ distribution parameter estimation method has been proposed. The proposed method successfully solves the problems that "the MoM cannot realize the full range estimation of $G^{0}$ distribution parameters" and "the MLE method needs a long iterative time and has the poor iteration accuracy." More importantly, the method considers the ENL parameter as an unknown estimated parameter like the shape and scale parameters, which can ensure that $G^{0}$ distribution has higher fitting precision. The method not only has the advantage of the SKS method that can quickly and accurately estimate the parameters of $G^{0}$ distribution but also overcomes the disadvantage of both SKS method and MoM method that are not applicable when the clutters are very homogeneous. Experimental results verify the effectiveness 


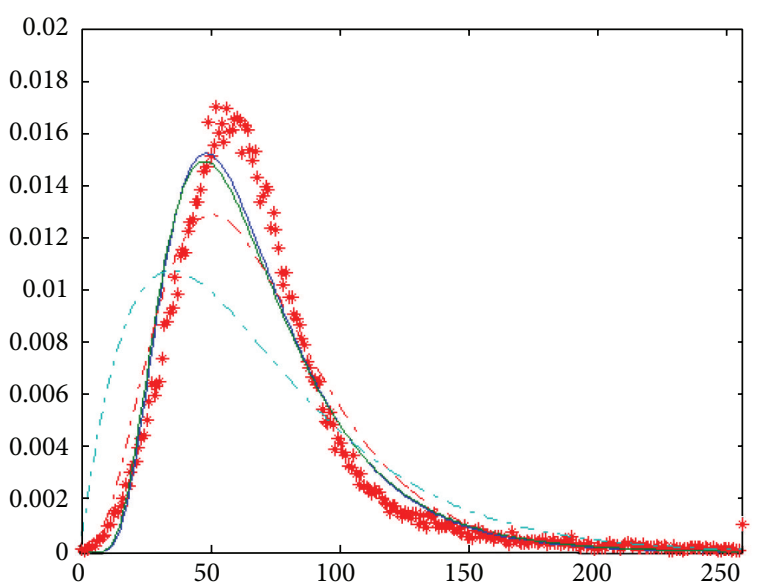

* Histogram

- MoM-based lognormal

- MLE-based lognormal

-. - MoM-based gamma

MLE-based gamma

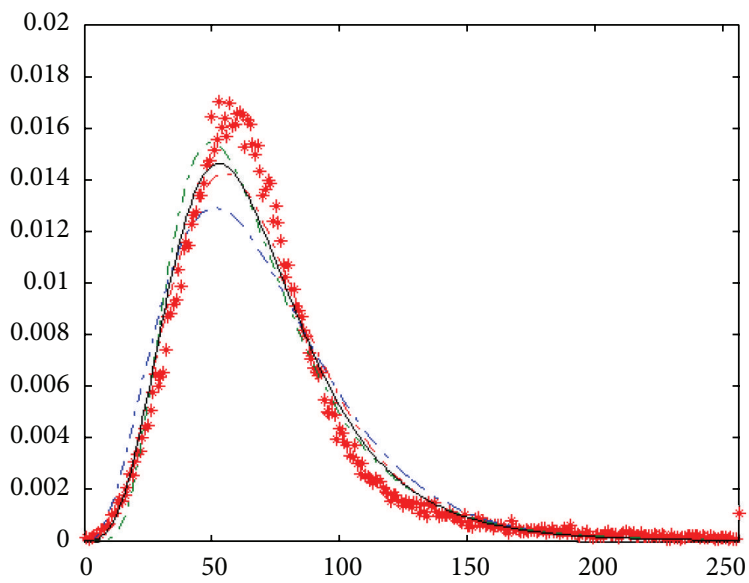

* Histogram

-. - Freitas' MoM-based $G^{0}$

-. - Fully parametric MoM

...- SKS-based $G^{0}$

— SKS + MoM-based $G^{0}$

Figure 8: The fitting results of Region 6.

of the proposed method in the SAR image background clutter modeling. The clutter statistic model based on the proposed method can be widely used for the SAR image interpretation such as SAR target detection and discrimination in the future research.

\section{Appendix}

\section{Description}

In the expressions in Tables 4(a) and 4(b), $x$ and $x_{i}$ denote the intensity or amplitude of pixels. $\mu$ and $\sigma^{2}$ denote the mean and variance of image pixel intensity or amplitude, respectively. $N$ denotes the total number of pixels that participate in the operation. $\Gamma(x)$ is called the gamma function,
$\psi(x)=\Gamma^{\prime}(x) / \Gamma(x)$ is called the digamma function, and $K_{v}(x)$ is called the $v$-order $K$-class Bessel function. $n$ is the equivalent number of looks, $\alpha$ is the shape parameter, and $\gamma$ is the scale parameter of $G^{0}$ distribution. $\widetilde{c}_{1}$ is the first-order sample log-cumulant.

\section{Conflict of Interests}

The authors declare that there is no conflict of interests regarding the publication of this paper.

\section{Acknowledgments}

The authors are grateful to the anonymous referees for their valuable comments and suggestions. This research is partially supported by the National Natural Science Foundation of China (61105020) and Key Research Platform for Research Projects (13zxtk08).

\section{References}

[1] C. C. Freitas, A. C. Frery, and A. H. Correia, "The polarimetric $\mathscr{G}$ distribution for SAR data analysis," Environmetries, vol. 16, no. 1, pp. 13-31, 2005.

[2] T. Eltoft, "Modeling the amplitude statistics of ultrasonic images," IEEE Transactions on Medical Imaging, vol. 25, no. 2, pp. 229-240, 2006.

[3] M. S. Greco and F. Gini, "Statistical analysis of high-resolution SAR ground clutter data," IEEE Transactions on Geoscience and Remote Sensing, vol. 45, no. 3, pp. 566-575, 2007.

[4] C. Tison, J.-M. Nicolas, F. Tupin, and H. Maître, "A new statistical model for Markovian classification of urban areas in high-resolution SAR images," IEEE Transactions on Geoscience and Remote Sensing, vol. 42, no. 10, pp. 2046-2057, 2004.

[5] J. M. Nicolas and F. Tupin, "Gamma mixture modeled with 'second kind statistics': application to SAR image processing," in Proceedings of the IEEE International Geoscience and Remote Sensing Symposium (IGARSS '02), pp. 2489-2491, June 2002.

[6] R. Abdelfattah and J.-M. Nicolas, "Interferometric SAR coherence magnitude estimation using second kind statistics," IEEE Transactions on Geoscience and Remote Sensing, vol. 44, no. 7, pp. 1942-1953, 2006.

[7] Y. Li, S.-X. Wang, K.-F. Ji, and Y. Su, "A new method of automatic target discrimination in high-resolution SAR image," Journal of National University of Defense Technology, vol. 29, no. 3, pp. 8184, 2007.

[8] S. Kuttikkad and R. Chellappa, "Non-Gaussian CFAR techniques for target detection in high resolution SAR images," in Proceedings of the 1st International Conference on Image Processing, pp. 910-914, Austin, Tex, USA, 1994.

[9] J. S. Salazar and D. R. Hush, "Statistical modeling of target and clutter in single-look non-polorimetric SAR imagery," in Proceedings of the IASTED International Conference on Signal and Image Processing, pp. 272-276, Las Vegas, Nev, USA, October 1998.

[10] A. C. Frery, H.-J. Müller, C. D. C. F. Yanasse, and S. J. S. Sant'Anna, "A model for extremely heterogeneous clutter," IEEE Transactions on Geoscience and Remote Sensing, vol. 35, no. 3, pp. 648-659, 1997. 
[11] G. Gao, M. Lu, J. J. Huang, G. Y. Kuang, and D. R. Li, "Statistical analysis of clutter in high-resolution SAR images," Signal Processing, vol. 24, no. 4, pp. 648-654, 2008.

[12] C. H. Gierull and I. C. Sikaneta, "Estimating the effective number of looks in interferometric SAR data," IEEE Transactions on Geoscience and Remote Sensing, vol. 40, no. 8, pp. 1733-1742, 2002.

[13] W. Z. Li, X. S. Ku, J. H. Cheng, and J. C. Wang, "A new $G^{0}$ distribution parameter estimation method for SAR images," in Proceedings of the 11th National Radar Academic Conference, pp. 210-214, November 2010.

[14] G. T. Shi, G. Gao, X. G. Zhou, G. Y. Kuang, and Y. M. Jiang, "The Mellin transform-based $\mathrm{G}^{0}$ distribution parameter estimation method," Progress in Natural Science, vol. 19, no. 6, pp. 677-684, June 2009.

[15] K. Huangfu, J. W. Chen, S. Q. Lou et al., Modern Digital Signal Processing, chapter 6, Publishing House of Electronics Industry, Beijing, China, 2012.

[16] L. X. Wang, D. Z. Fang, M. Y. Zang et al., Handbook of Mathematics, chapter 5, Higher Education Press, Beijing, China, 1979.

[17] G. Franceschetti and R. Lanari, Synthetic Aperture Radar Processing, chapter 1, CRC Press, Rome, Italy, 1999.

[18] G. Gao, The research on automatic acquirement of target's ROI from SAR imagery [Ph.D. thesis], National University of Defense Technology, Changsha, China, 2007.

[19] M. D. DcVore and J. A. O'Sullivan, "Statistical assessment of model fit for synthetic aperture radar data," in Algorithms for Synthetic Aperture Radar Imagery VIII, vol. 4382 of Proceedings of SPIE, Orlando, Fla, USA, April 2001. 




Advances in

Operations Research

mansans



The Scientific World Journal
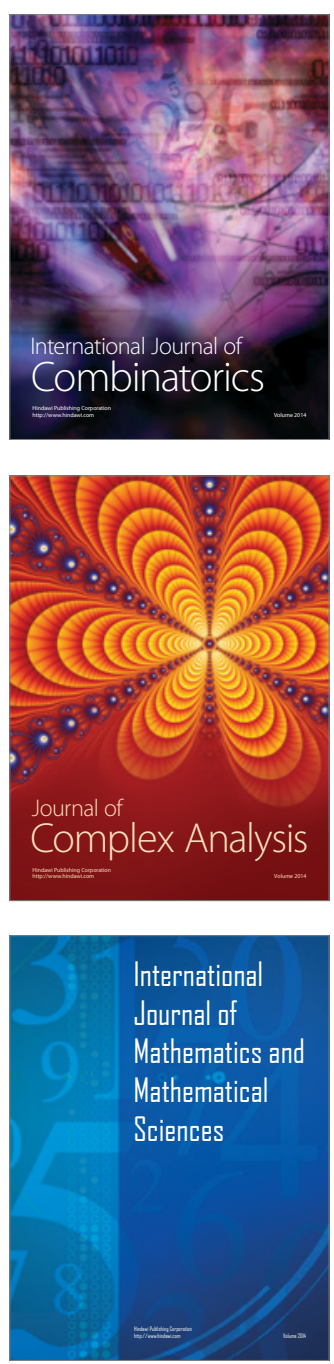
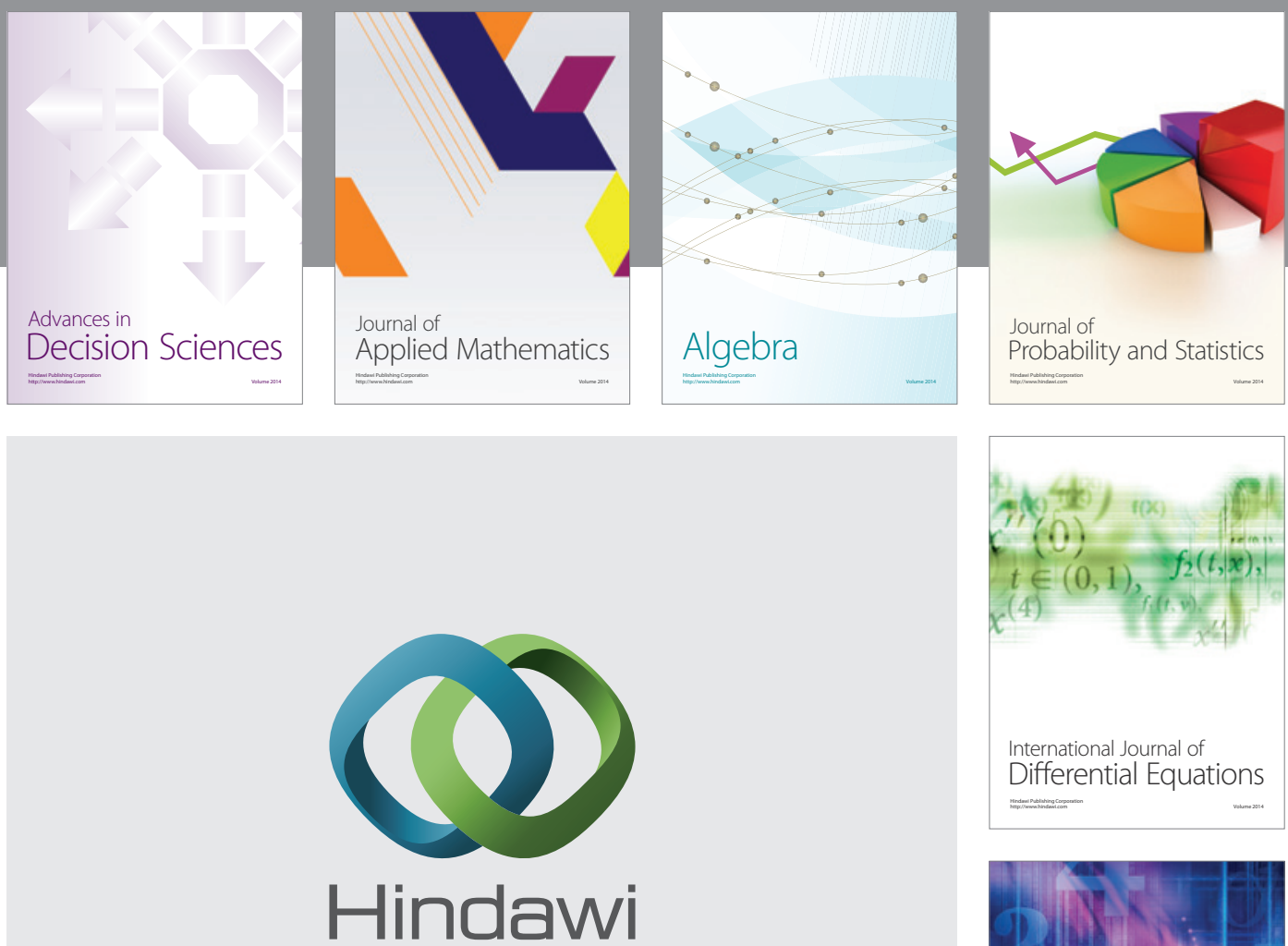

Submit your manuscripts at http://www.hindawi.com
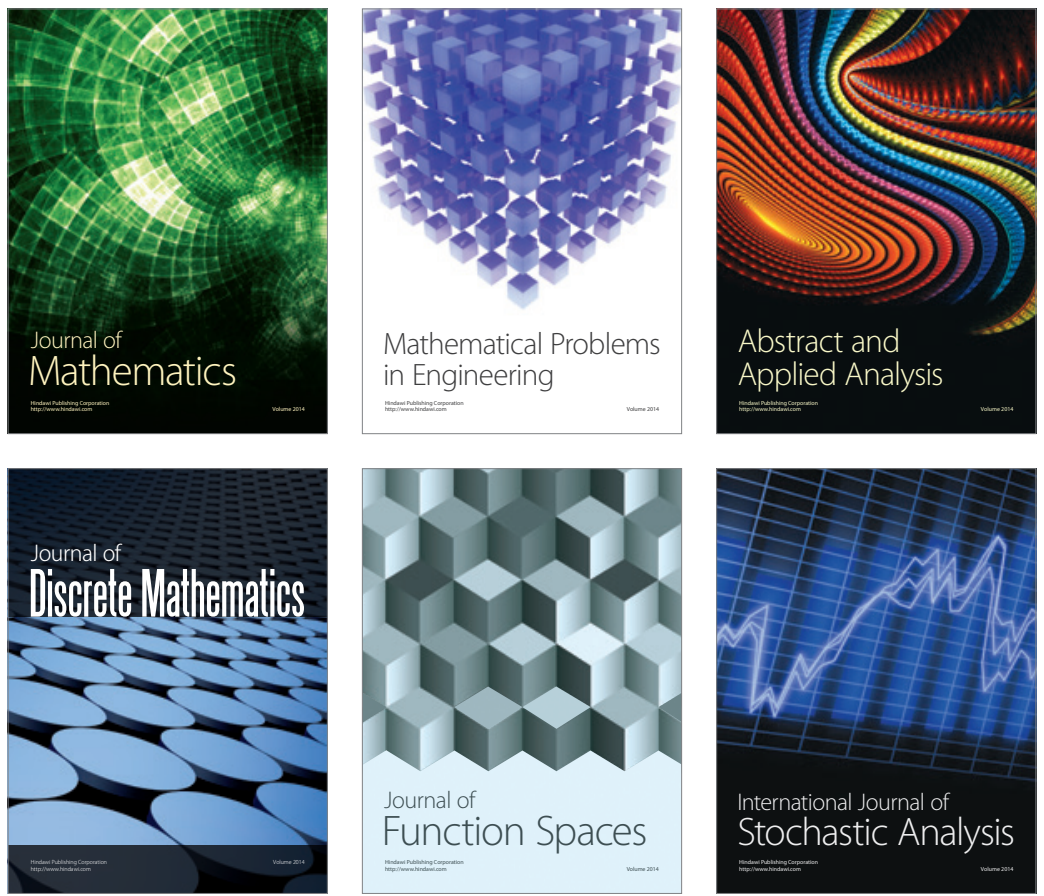

Journal of

Function Spaces

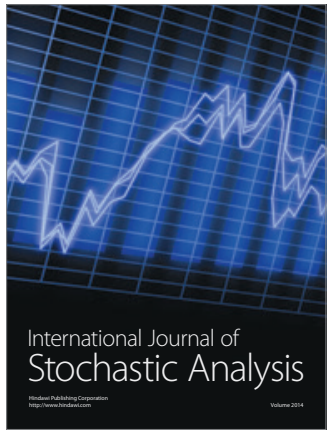

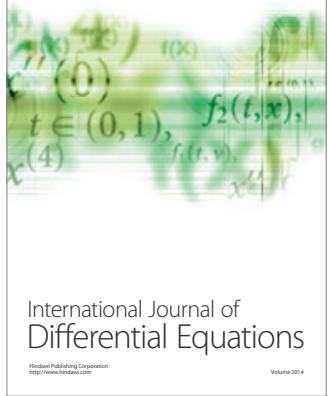
